\title{
STABLE FACES OF A POLYTOPE
}

\author{
BY GOTTFRIED T. RÜTTIMANN ${ }^{1}$
}

Communicated by Robert Bartle, December 1, 1975

The purpose of this announcement is to provide several new concepts for the study of the face-lattice of a polytope. The proofs of the results stated herein will appear elsewhere.

1. Preliminaries. Let $P$ be a polytope with $\operatorname{dim} P \geqslant 0$ [4].

Definition 1. A hyperplane $S$ in aff $P$ is said to be a support if $P$ is entirely contained in one of the closed halfspaces determined by $S .\{F(P), \leqslant\}$ denotes the poset of faces of $P$ which is indeed a lattice $(\Lambda$ : infimum, $\vee$ : supremum; 1: greatest element; 0: least element). $A \subseteq F(P)$ is called an order ideal if $a \leqslant b$ and $b \in A$ implies $a \in A$. The ideal [0,a] $(=\{b \in F(P) \mid 0 \leqslant b \leqslant a\})$ is called a principal order ideal (generated by $a$ ). A proper face $a$ is called split [1] if $\{b \mid b \wedge a=0\}$ is a principal order ideal and $P$ is the direct convex sum of $a$ and the generator of the primcipal order ideal. The faces 0 and 1 are called split.

$A$ face of $P$ is split if and only if it is a central element of the face-lattice.

Definition 2. A pair of faces $a, b \in F(P)-\{1\}$ is said to be orthogonal, in symbols $a \perp b$, provided there exist two supports $S_{1}, S_{2}$ such that $a \subseteq S_{1}, b$ $\subseteq S_{2}$ and $S_{1} \cap S_{2}=\varnothing$. We put: $a \perp 1(1 \perp a)$ if and only if $a=0$. If $A \subseteq$ $F(P)$, define $A^{\perp}=\{b \in F(P) \mid b \perp a$ for all $a \in A\}$. If $\{a\}^{\perp}$ is a principal order ideal, then $a^{\perp}$ denotes its generator.

We have immediately: (i) $a \perp b \Rightarrow b \perp a$, (ii) $0 \perp a$ for all $a \in F(P)$, (iii) $a \perp a \Longleftrightarrow a=0$, (iv) $a \perp b \Rightarrow a \wedge b=0$, (v) $a \leqslant b$ and $b \perp c \Rightarrow a \perp c$.

\section{Mutually stabilizing pairs of faces.}

Definition 3. A face $a \in F(P)$ is said to be stable provided there exists a face $b$ such that $a$ is a maximal element in the subposet $\left\{\{b\}^{\perp}, \leqslant\right\} . \quad S(P)$ denotes the set of stable faces of $P$. A pair $a, b \in F(P)$ is called mutually stabilizing in symbols $a \Perp b$, if $a$ is a maximal element in $\left\{\{b\}^{\perp}, \leqslant\right\}$ and $b$ is a maximal element in $\left\{\{a\}^{\perp}, \leqslant\right\}$.

Note that facets and split faces are stable. One easily gets: (i) $a \Perp b \Rightarrow$

AMS (MOS) subject classifications (1970). Primary 52A25, 06A10; Secondary 81A12.

Key words and phrases. Stable face, split face, strong polytope, simplex, orthomodular poset, Boolean lattice, pure state, dispersion-free state, superposition of states.

${ }^{1}$ Research supported by the Swiss National Science Foundation 
$b \Perp a$, (ii) $a \Perp b \Rightarrow a \perp b$, (iii) $a \Perp b \Rightarrow a, b \in S(P)$, (iv) $0 \Perp a \Longleftrightarrow a=1,1 \Perp$ $a \Longleftrightarrow a=0$.

THEOREM 1. For every stable face a of a polytope there exists at least one face $b$ such that the pair $a, b$ is mutually stabilizing.

THEOREM 2. If $a \Perp b(a, b \neq 1)$ then there exists exactly one pair of supports $S_{1}, S_{2}$ such that $a \subseteq S_{1}, b \subseteq S_{2}$ and $S_{1} \cap S_{2}=\varnothing$.

THEOREM 3. $a \Perp b$ if and only if $a \perp b$ and $\operatorname{dim}(a \cup b)=\operatorname{dim} P$.

COROLlaRY 4. $a \in F(P)$ is a stable face if and only if there exists a face $b$ such that $a \perp b$ and $\operatorname{dim}(a \cup b)=\operatorname{dim} P$.

\section{Strong polytopes.}

Definition 4. A polytope $P$ is said to be strong provided (i) if $a \in S(P)$ then $\{a\}^{\perp}$ is a principal order ideal in $F(P)$, (ii) if $a \perp b, a \# b$ where $a, b \in$ $S(P)$ then $\{a, b\}^{\perp \perp}$ is a principal order ideal in $F(P)-\{1\}$.

If $P$ is a strong polytope then $a \Perp b \Longleftrightarrow a^{\perp}=b(a \in S(P), b \in F(P))$, and therefore $S(P)$ is closed under the mapping $a \rightarrow a^{\perp}$.

THEOREM 5. If $P$ is a strong polytope then $\left\{S(P), \leqslant,{ }^{\perp}\right\}$ is an orthomodular poset. Its center coincides with the set of split faces. $\left\{S(P), \leqslant,{ }^{\perp}\right\}$ is an orthomodular lattice if and only if $\{a \vee b\}^{\perp}$ is a principle order ideal in $F(P)$ for all $a, b \in S(P)$.

(For definitions and basic properties of orthomodular posets see [3].)

A simplex is a strong polytope and $S(P)=F(P)=$ \{split faces $\}$. The orthomodular poset $\left\{S(P), \leqslant^{\perp}\right\}$ is Boolean (i.e.: a Boolean lattice).

THEOREM 6. Let $P$ be a strong polytope. If the orthomodular poset $\left\{S(P), \leqslant,{ }^{\perp}\right\}$ is Boolean then $P$ is a simplex.

4. States. The issue of this section is to show that with each point or a strong polytope $P$ we can associate in a unique manner a state (probability measure) [2] on the orthomodular poset of stable faces. The treatment is purely geometrical.

Let $P$ be a polytope with $\operatorname{dim} P \geqslant 1, S_{1}, S_{2}$ supports such that $S_{1} \cap S_{2}$ $=\varnothing$ and $\nu \in$ aff $P$. Then there exist elements $\nu_{1} \in S_{1}, \nu_{2} \in S_{2}$ and a unique real number $\mu\left(\nu, S_{1}, S_{2}\right)$ such that

$$
\nu=\mu\left(\nu, S_{1}, S_{2}\right) \nu_{1}+\left(1-\mu\left(\nu, S_{1}, S_{2}\right)\right) \nu_{2} .
$$

Now let $P$ be a strong polytope $(\operatorname{dim} P \geqslant 0)$. For all $\omega \in P$ and $a \in S(P)$ we define

$$
\mu_{\omega}(a)= \begin{cases}0 & \text { if } a=0, \\ 1 & \text { if } a=1, \\ \mu\left(\omega, S_{1}, S_{2}\right) & \text { if } a \neq 0,1\end{cases}
$$


where $S_{1}, S_{2}$ is the unique pair of supports such that $a \subseteq S_{1}, a^{\perp} \subseteq S_{2}$ and $S_{1} \cap$ $S_{2}=\varnothing\left(\right.$ see Theorem 2). Note that $0 \leqslant \mu_{\omega}(a) \leqslant 1$ for all $\omega \in P$ and $a \in S(P)$. Denote $\Delta=\left\{\mu_{\omega} \mid \omega \in P\right\}$.

THEOREM 7. For every $\omega \in P$ the mapping $a \in S(P) \rightarrow \mu_{\omega}(a) \in[0,1]$ is a state on the orthomodular poset $\left\{S(P), \leqslant,{ }^{\perp}\right\}$. The mapping $\omega \in P \rightarrow \mu_{\omega}$ $\in \Delta$ is one-to-one.

THEOREM 8. Let $P$ be a strong polytope. Then

(i) $\Delta$ is a strongly order determining set of states for the orthomodular poset of stable faces;

(ii) $\mu_{\omega}$ is a pure state (with respect to $\Delta$ ) if and only if $\omega \in \operatorname{ext} P$;

(iii) $\mu_{\omega}$ is a dispersion-free state if and only if, for all $a \in S(P), \omega \notin a$ implies $\omega \in a^{\perp}$;

(iv) $\mu_{\omega}$ is a superposition [5] of a family of states $\left\{\mu_{\omega_{i}} \mid i \in I\right\}$ if and only if $\omega$ belongs to the face generated by $\left\{\omega_{i} \mid i \in I\right\}$.

5. Remark. In a subsequent paper we will give a characterization of those orthomodular posets that are ortho-order isomorphic to the orthomodular poset of stable faces of some strong polytope. The key notion in that investigation is a generalized version of the Jordan-Hahn decomposition of signed measures.

\section{REFERENCES}

1. E. M. Alfsen, Compact convex sets and boundary integrals, Springer-Verlag, Berlin and New York, 1971.

2. M. K. Bennett, States on orthomodular lattices, Natur. Sci. and Math. 8 (1968), 47-51. MR 38 \#88.

3. D. J. Foulis, $A$ note on orthomodular lattices, Portugal. Math. 21 (1962), 65-72. MR 26 \#6088.

4. B. Grünbaum, Convex polytopes, Pure and Appl. Math., vol. 16, Interscience, New York, 1967. MR 37 \#2085. 1968.

5. V. S. Varadarajan, Geometry of quantum theory, Van Nostrand, Princeton, N. J.,

DEPARTMENT OF MATHEMATICS, UNIVERSITY OF MASSACHUSETTS, AMHERST, MASSACHUSETTS 01002 